\title{
Clarifying brain structure, literally
}

A fluorescence-compatible tissue-clearing reagent enables light microscopy-based imaging deep in the mouse brain .

In The Invisible Man, a science fiction novella by Herbert G. Wells, the protagonist is a scientist who finds a way to make the human body invisible by changing its refractive index to prevent the bending and reflection of light. In a recent report, Atsushi Miyawaki and his colleagues at RIKEN described the development of a tissue-clearing reagent with similar effects, bridging the gap between science and fiction and enabling fluorescence-based imaging of biological tissues at unprecedented depth and subcellular resolution.

High-resolution microscopy methods and fluorescence-based labeling techniques have enabled the three-dimensional imaging and reconstruction of defined cellular populations in a variety of biological tissues. However, axial resolution and imaging depth are often limited by the intrinsic opacity of biological specimens. For example, in visualizing the mammalian brain, light microscopy-based advances have been confined to the few hundred micrometers under the organ's surface. Alternatively, mechanical sectioning or insertion of minuscule endoscopes can be used to access deeper structures, but such approaches are inevitably laborious, invasive or of limited perspective.

According to Miyawaki, tissue opacity stems predominantly from light scattering rather than absorption, so "if we can get rid of the scattering, we can make the tissue transparent" and thus increase the imaging depth. Several tissue-clearing reagents have been reported previously but these are either prohibitively expensive and of proprietary composition, or inefficient and poorly compatible with fluorescence-based microscopy.

To overcome these setbacks, Miyawaki and colleagues developed a tissue-clearing solution, Scale, superior to alternative techniques in terms of both cost and performance. The aqueous formula is amazingly simple and consists of inexpensive compounds commonly found in research laboratories. According to Miyawaki, Scale renders biological tissues almost completely transparent by "eliminating the scattering of light, without affecting absorption at all" and is mild enough to preserve the spectral properties of most protein-based and chemical fluorescent labels. In addition, researchers can modify

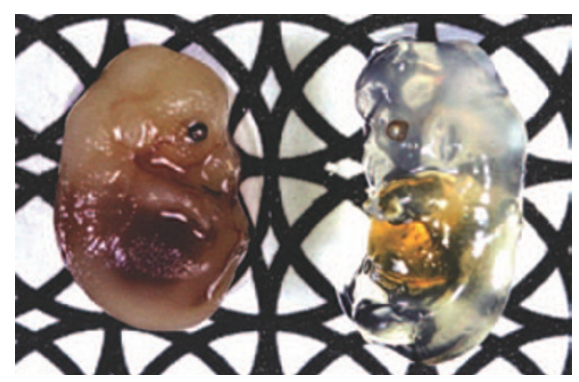

Tissue clearing. A Scale-clarified mouse embryo (right) compared to a control embryo (left) against a patterned background. Reprinted from Nature Neuroscience.

the solution according to the tissue of interest or if necessary to minimize some of the method's shortcomings, such as the need for prolonged sample incubation or the associated tissue expansion.

The combined properties of Scale make possible the visualization of fluorescently labeled mouse brain samples at unprecedented depths, limited solely by the working distance of the objective lens. Using customdesigned optics on Scale-cleared samples, Miyawaki and colleagues reconstructed the three-dimensional architecture of millimeter-scale neural networks spreading the cerebral cortex, white matter and hippocampal regions. In a neurogenic niche of the adult brain, the researchers visualized proliferating neural stem cells and their close association with the surrounding cerebral vasculature in astonishing detail. Combining Scale with a cell cycle-specific labeling approach provided a comprehensive perspective of proliferation versus differentiation at various stages of embryonic development.

Although the group's current work is focused on the application of Scale to various aspects of brain structure and development, the technique should be readily extendable to a variety of fixed soft tissues, including human biopsy samples, explains Miyawaki. In an optimistic manner, he alludes to the ongoing development of milder reagents, which might one day allow deeper imaging in live biological specimens.

\section{Petya V Krasteva}

\section{RESEARCH PAPERS}

Hama, H. et al. Scale: a chemical approach for fluorescence imaging and reconstruction of transparent mouse brain. Nat. Neurosci. advance online publication (30 August 2011). 